Validation of the Sexual Communication Self-Efficacy Scale 


\begin{abstract}
This study assessed a newly developed Sexual Communication Self-Efficacy Scale (SCSES) designed to measure the sexual communication self-efficacy of adolescent men and women. Three-hundred and seventy-four U.K. adolescents completed this new scale, along with several other validity measures. Factor analysis revealed that the SCSES consisted of 5 underlying factors: contraception communication, positive sexual messages, negative sexual messages, sexual history, and condom negotiation. These factors demonstrated high internal consistency, and presents evidence to support construct validity. This scale may have utility in assessing the effectiveness of interventions designed to enhance sexual communication and sexual health behaviors among young people.
\end{abstract}

Keywords: adolescence, sexual health, sexual communication self-efficacy 


\section{Validation of the Sexual Communication Self-Efficacy Scale}

\section{Introduction}

Many adolescents engage in sexual intercourse in the US (Eaton et al., 2012), and in the UK (Mercer et al., 2013), and unsafe sex is one of the main risk factors for young people between 10-24 years globally (Gore et al., 2011). The prevalence of sexual risk taking among adolescents is high, and young people aged $16-24$ years in the UK are most at risk for STIs (Health Protection Agency, 2008), and are more likely to report not using condoms with at least two sexual partners in the last year (Mercer et al., 2013). Further, the highest prevalence of chlamydia in the UK was among women aged 18-19 years and men aged 20-24 years (Sonnenberg et al., 2013). Meanwhile, many UK adolescents (21-22\%) indicate that they did not use condoms during their first intercourse experience (Wellings et al., 2001) and a high proportion of 16-24 year old UK men (43.8\%) and women (51.9\%) lacked sexual competence at first sexual intercourse (e.g., lacking decision autonomy, lacking acceptable timing, and not using a reliable contraceptive method; Wellings, 2013). Poor verbal communication between partners may be a significant factor contributing to adolescent risk taking. Adolescents who communicate more frequently with their partners about HIV, pregnancy, condom use and other contraceptive use issues are more likely to use contraception consistently (Davies et al., 2006).

Sexual communication is commonly highlighted as a key factor influencing sexual health behavior and, in particular, condom use. Research by Troth and Peterson (2000) found that adolescents who have positive attitudes about safer sex communication use condoms more often. Additionally, Grossman (2008) found that more frequent discussions of condom use predicted consistent condom use and, likewise, that less frequent discussions about condoms was predictive of inconsistent condom use. It is also important to consider adolescent sexuality 
broadly to include positive aspects of adolescent sexual well-being, and not prioritize only the absence of disease or the limiting of risk behaviors. Instead, this broader conceptualization also privileges the development of a positive and healthy sexuality, and considers adolescents' involvement in sexual relationships as a normative transition (Tolman, Striepe, \& Harmon, 2003). Interestingly, encouraging positivity about sexuality may have important implications for sexual health among this age group, through increasing the effectiveness of HIV/STI and pregnancy prevention interventions (Philpott, Knerr, \& Maher, 2006), and helping to facilitate the information that adolescents need to have enjoyable and fulfilling sexual lives (Ivankovich, Leichliter, \& Douglas, 2013; Wellings \& Johnson, 2013;). Among undergraduate men and women, those reporting a more loving first sexual experience were not only more likely to rate the experience as positive, but also were more likely to report using protection, and to be in their relationships longer before having intercourse (Smiler, Ward, Caruthers, \& Merriwether, 2005). In addition, good quality communication with one's partner is associated with positive outcomes such as relationship and sexual satisfaction (Byers, 2005), and general sexual communication (e.g., communicating about fantasies and desires) has benefits for contraceptive use beyond contraceptive communication alone (Widman, Welsh, McNulty, \& Little, 2006).

Yet, adolescents often have difficulty discussing issues like safe sex with their partners (Guzman et al., 2003). Self-efficacy, beliefs about one's ability to engage in a desired behavior or achieve a level of performance (Bandura, 1994), may be a key factor in adolescent sexual communication. Self-efficacy is a commonly assessed construct in adolescent sexual health research (Pearson, 2006; Rostosky, Dekhtvar, Cupp, \& Anderman, 2008), and self-efficacy to communicate is associated with more positive condom attitudes, and more condom use (HalpernFelscher et al., 2004). There are a number of existing scales which measure aspects of 
communication within sexual relationships. Some scales focus specifically on parent-adolescent sexual communication (e.g., Miller, Kotchik, Dorsey, Forehand, \& Ham, 1998), while others assess sexual communication frequency (e.g., Sales et al, 2008). Other studies also measured sexual communication self-efficacy, but did so with single item measures (e.g., Halpern-Felscher et al., 2004), or with a distinct focus on managing risky sexual behaviors and resisting unwanted sex (e.g., Rostosky, Dekhtvar, Cupp, \& Anderman, 2008). Importantly, these scales focus primarily on risk reduction, and do not approach the topic from a perspective that also considers communicating about positive topics such as interest in sexual behaviors. The scale developed in the present study expands on these scales and presents a valid and reliable instrument for assessing sexual communication self-efficacy between partners which also incorporates both positive and risk-related sexual communication topics.

\section{The Present Study}

Given the potentially important role of sexual communication self-efficacy to protective sexual behaviors, such as condom and hormonal contraceptive use, and to relationship and sexual satisfaction, there is a need for a valid and reliable measure of sexual communication selfefficacy. This study examines the newly created Sexual Communication Self-Efficacy Scale for adolescents, which assesses confidence discussing sexual health topics (such as condom use and sexually transmitted infections), sexual pleasure topics (such as indicating when an activity feels good) as well as sexual limits (such as indicating one does not want to have sex). This paper reports on the validation of this scale, including factor analysis, validity, and internal reliability.

\section{Methods}

\section{Participants}


The sample consisted of 374 U.K. adolescents, most were female ( $\mathrm{n}=216 ; 59 \%)$; but a substantial percentage were male $(n=158 ; 42 \%)$. Ages ranged from 16 to 22 year olds $(M=$ $18.29, S D=1.35)$, most of whom were enrolled at a college or university $(64.2 \%)$, while $13.9 \%$ were currently in high school, and $9.4 \%$ were working. Most of the participants were White (73\%), while the other notable proportions were Black (3\%) and of mixed cultural background (6\%). In addition, a substantial number of participants were in a relationship (56.4\%). Fortytwo percent of the sample indicated that the last time they had sex was less than a week ago, and $26 \%$ indicated that the last time they had sex was one to four weeks ago.

\section{Procedure}

Participants were recruited through advertisements on Facebook. After clicking the advertisement, users were presented with the "Sexunzipped" website survey enrolment page, and with an eligibility questionnaire. If they indicated that they were residents of the UK and between the ages of 16 and 22, they were presented with introductory information explaining the purpose of the study (i.e., to examine sex, relationships, and health). Participants were informed that their answers would help the researchers design a website about sexual health for young people, and that it would take approximately 30 minutes to complete. After providing basic demographic information for an eligibility check, including their age, gender, location, and whether they had ever had intercourse, they were presented with a consent form. First, they were asked to consent that they understood the nature of the study, that they consented to fill in questionnaires about sex and relationships, and that they would give their postal address in order to collect their $£ 10$ (\$16) voucher. On registration, participants were asked to provide first and last name, and postal and email address, and were automatically assigned an ID number to ensure that the data could be anonymised. After completing the survey, participants were given 
a debriefing form, which included web links and information for help lines for sexual and domestic abuse.

\section{Measures}

Sexual Communication Self-Efficacy Scale. The Sexual Communication Self-Efficacy Scale consisted of 22 items that measured respondents' confidence in engaging in a variety of activities with a sexual partner along a 4-point likert scale ( $1=$ Very Difficult, $4=$ Very Easy). Several of the Sexual Communication Self-Efficacy Scale (SCSES) items were developed as part of an NIMH funded HIV intervention grant by two of the authors (Names not included for anonymity of manuscript). Items were developed based on a review of the literature and consultations with sexual health educators to assess six sexual risk-related areas (e.g., IV drug use, STI history), and then reviewed in focus groups with African American adolescents women to determine their relevance and phrasing. Eighteen items were developed in the initial pool, and pilot testing reduced these to 7 (these 7 items are identified with an asterix in Table 2). For this project, additional Sexual Communication Self-Efficacy scale items were developed to assess constructs not incorporated in the original measure (i.e., such as related to sexual pleasure, sexual negotiation). Interviews with 12 adolescents from London, U.K., were also conducted in order to ensure young people understood the meaning of the items. Based on feedback from these adolescents, 22 items were finalized and used in subsequent analyses. Factor analysis conducted in this study provided support for 5 factors comprised of 20 items: Contraception Communication (e.g., "Discuss contraception?”), Positive Sexual Messages (e.g., “Tell them you want to have sex more often?"), Negative Sexual Messages (e.g., "Tell them that a sexual activity hurts you?"), Sexual History (e.g., “Ask if they have shared needles?”), and Condom Negotiation (e.g., "Demand that a condom be used?"). The current analysis indicates that internal 
consistency of all factors was strong, and Cronbach's alpha coefficients are: contraceptive communication $(\alpha=.89)$, negative sexual messages $(\alpha=.87)$; positive sexual messages ( $\alpha=$ $.88)$; sexual history $(\alpha=.82)$; condom negotiation $(\alpha=.83)$.

Sexual Communication Frequency. Frequency of sexual communication was assessed with 6 items prefaced with "Have you talked about these things with current or most recent partner/s? followed by five statements: (1) The kind of sex you like; (2) The kind of sex your partner likes; (3) Sexually transmitted infections; (4) Using condoms; (5) Using contraception (birth control); (6) Pregnancy. Response choices were 0 (yes) and 1 (no). Scores were summed, with total scores ranging from 0 to 6 . High scores indicate lower sexual communication frequency. Cronbach's alpha for this measure was .74.

Dyadic Sexual Communication. Dyadic communication was assessed by a 6-item scale developed by Catania, Coates, and Kegeles, 1989. The scale consisted of the following six statements: (1) My partner rarely responds when I want to talk about our sex life; (2) Some sexual matters are too upsetting to discuss with my sexual partner; (3) There are sexual issues or problems in our sexual relationship that we have never discussed; (4) My partner has no difficulty in talking to me about his or her sexual feelings and desires; (5) Talking about sex is a satisfying experience for both of us; (6) I have little difficulty in telling my partner what I do or don't do sexually. Participants responded on a 5-point Likert-type rating scale, with responses ranging from 1 (strongly agree) to 5 (strongly disagree). The last 3 questions were reversescored. The total score was calculated by the mean of the items, with scores ranging from 1 to 5 . High scores indicate high dyadic sexual communication. Cronbach's alpha for this measure was .70 . 
Communication Intentions. Communication intention was examined with 6 questions measured along a 5-point Likert scale ( $1=$ Definitely not, $5=$ Definitely $)$. Questions asked about the individual's intention to use contraception with a future partner (e.g., "Use a condom if you have vaginal sex with a new partner"), intention to have an STI test with a new partner (e.g., "Have test for sexually transmitted infections if you have a new partner"), and discussing sexual pleasure (e.g., “discuss sexual enjoyment with partner(s)?”). The total score was calculated for these items, with possible scores ranging from 9 to 30. Cronbach's alpha in this study was .74.

Sexual Self-Awareness. Sexual self-awareness was assessed with 3 items consisting of the following three statements: (1) I know what turns me on; (2) I know what I want when it comes to sex; (3) I know what I want when it comes to relationships. Participants responded on a 5-point Likert-type rating scale, with responses ranging from 1 (strongly agree) to 5 (strongly disagree). The total score was calculated by the mean of the items, with scores ranging from 1 to 5. High scores indicate lower sexual self-awareness. Cronbach's alpha for the scale was .63.

Sexual Pressure. Sexual pressure was assessed by 3 items which asked if in the last 3 months the participant had been (1) Pressured into kissing or touching; (2) Pressured into oral sex; (3) Pressured into having sexual intercourse. Response choices were 0 (yes), 1 (not sure) and $2(n o)$. Total scores were summed, with scores ranging from 0 to 6 . High scores indicate lower pressure. Cronbach's alpha for the scale was .66.

Relationship Quality. Relationship quality was assessed with 5-items: (1) I enjoy the time we spend together; (2) I enjoy our physical contact; (3) I can confide in them (tell them private things); (4) My partner respects me; (5) My partner is honest with me. Participants responded on a 5-point Likert-type rating scale, with responses ranging from 1 (strongly agree) 
to 5 (strongly disagree). Total scores were summed, with scores ranging from 5 to 25 . High scores indicate lower relationship quality. Cronbach's alpha for this measure was .77.

Intimate Partner Abuse. The HARK (Humiliation, Afraid, Rape, Kick) tool by Sohal, Eldridge, and Feder (2007) consists of 4 items. Originally, the scale asked about experiencing abuse in the last year, but the scale was modified for the current project to ask if in the last 3 months a participant has been (1) Humiliated or emotionally abused in other ways by a partner or ex-partner; (2) Afraid of a partner or ex-partner; (3) Forced to have any kind of sexual activity by a partner or ex-partner; (4) Kicked, hit, slapped or otherwise physically hurt by a partner or expartner. Response choices were 0 (yes), 1 (not sure) and 2 (no). The total score was calculated by the mean of the items, with scores ranging from 0 to 3 . Higher scores indicate intimate partner abuse. Cronbach's alpha for this measure was .64.

Condom Use Self-Efficacy. Condom use self-efficacy was examined with 9 questions along a 4-point Likert scale ( 1 = I definitely could, 4 = I definitely could not). Questions ask about confidence obtaining condoms (e.g., "Get condoms if you need them?"), and condom use (e.g., "Put a condom on correctly?"). Score were summed to create a total, with possible scores ranging from 9 to 36 . Cronbach's alpha in this study was .73 .

Analysis. Sexual communication self-efficacy items were subjected to a maximum likelihood factor analysis in SPSS 20, and following procedures detailed by Tabachnick and Fidell (2012). Factors were assumed to be correlated and therefore Oblimin rotation was used. Following this, internal consistency for items loading on a factor was assessed using Cronbach's alpha. Subscale scores were calculated by taking the mean of items loading on a factor, and means and standard deviations for each subscale were calculated. Items were subjected to a Flesch-Kincaid Reading Ease calculator. Construct validity was assessed by correlating subscale 
scores with variables assumed to be conceptually related including sexual communication frequency, dyadic sexual communication, communication intentions, sexual self-awareness, sexual pressure, relationship quality, interpersonal violence, and condom use self-efficacy.

\section{Results}

\section{Factor Analysis}

An initial analysis indicated, based on eigenvalues, that a 5 factor solution best fit the data. The scree plot suggested that either a 4 or 5 factor solution would best fit the data. Given the large sample size, and the cohesion of the themes underlying each factor in the 5 factor solution, the 5 factor solution was retained. Two items (items 15 and 16) loaded at around .40 on multiple factors, so they were excluded and the factor analysis was conducted once again, resulting in a final factor solution of 20 items with communalities ranging from .35 to .82 . The items loading onto each factor were thematically similar; factors were named Contraceptive Communication, Negative Sexual Messages, Positive Sexual Messages, Sexual History, and Condom Negotiation.

\section{Descriptive Statistics}

Means and standard deviations (Table 1) suggest that participants were most confident about communicating about aspects related to sexual history, and least confident communicating about condom negotiation. Overall, the adolescents of this study did not have high levels of communication self-efficacy related to any of the topics measured by the SCSES.

\section{Table 1 Here}


Response frequencies for the Sexual Communication Self-Efficacy Scale are shown in Table 2. Most participants indicated that they found it very difficult to request that a condom be used when having sex (57\%; item 5) and also found it very difficult to communicate that a sexual activity feels good to their partner (57\%; item 14).

\section{Table 2 Here}

\section{Internal Consistency of the SCSES}

The SCSES demonstrated high internal consistency. Cronbach's alphas for each subscale were high: (1) contraceptive communication $=.89 ;(2)$ negative sexual messages $=.87$; (3) positive sexual messages $=.88 ;(4)$ sexual history $=.82 ;(5)$ condom negotiation $=.83$. In addition, Cronbach's alpha for the scale with all items was .93 .

\section{Readability}

The Flesch-Kincaid assessment indicated that literacy grade level was 4.5. This specifies that a person would need to have reached between fourth and fifth grade to understand the language used. The Flesch Reading Ease score was 78.1 (scores range from 0 to 100, with higher scores indicating easier text to read).

\section{Construct Validity}

The SCSES was significantly correlated with all variables in expected directions (see Table 3). The strongest correlations were between Sexual Communication Self-Efficacy subscales and condom use self-efficacy. In addition, strong correlations between the SCSES subscales and the measure of Dyadic Sexual Communication were found, indicating positive evaluations with partner sexual communication were associated with greater confidence 
communicating about a range of sexual topics. All subscales of the SCSES were associated with increased sexual communication frequency, communication intentions, sexual self-awareness, relationship quality as well as lower levels of sexual pressure from their partner and interpersonal violence.

\section{Table 3 here}

\section{Discussion}

The purpose of this paper was to assess the newly created Sexual Communication SelfEfficacy Scale. This scale is the first to directly examine Sexual Communication Self-Efficacy, incorporating sexual health, sexual pleasure, and sexual negotiation items. Maximum likelihood factor analysis indicates that this scale is best represented by 5 factors, which we used to create subscales: Contraceptive Communication, Positive Sexual Messages, Negative Sexual Messages, Sexual History, and Condom Negotiation.

The results suggest that the SCSES is an internally consistent scale, as demonstrated by strong Cronbach's alpha coefficients across all 5 factors. As well, this study supports the construct validity of the scale, because of the demonstrated correlations between subscales and other measures. The associations found in this study are consistent with established literature on sexual communication as well as the limited research examining sexual communication selfefficacy. Specifically, this study mirrors other research which demonstrates that sexual communication self-efficacy is associated with condom attitudes (Halpern-Felscher et al., 2004). Unfortunately, the results of the present study also suggest that adolescent women from the current sample are, on average, not very confident communicating about any of the topics addressed in the Sexual Communication Self-Efficacy Scale. These results are similar to 
Guzman et al. (2003), who also found that sexual communication is problematic for adolescents. On the other hand, this study demonstrates the importance of communicating about sexual topics beyond sexual history and sexual risk. Participants in the current study who reported confidence communicating about positive sexual topics (i.e., that an activity feels good, that they wanted to have sex) reported higher relationship quality, more frequent and higher quality sexual communication, more self-awareness, and less interpersonal violence. They were also less likely to report experiencing sexual pressure, more likely to report intentions to communicate about sexual topics, and had greater confidence in their condom use ability.

Nonetheless, the findings of the current study are limited in several ways. First, despite the relatively large sample of young people, generalizability of these findings are limited because participants were a primarily white, adolescent, sample of UK residents. Additionally, most of the sample reported that they were in a relationship, thus it is not known if findings would generalize as well to those who would identify as single. In addition, several of the measures used to assess construct validity were not previously validated. However, these measures were developed based on a review of the literature, and field tested using qualitative think-aloud interviews with 12 young people to check meanings, and to help define appropriate response options. As a voluntary online study, self-selection bias is present. As a result, results may reflect beliefs and attitudes of young people with a more positive view of sexuality and more openness to responding to questions about sexuality and sexual health. However, it is dismaying in this case, that sexual communication self-efficacy with partners was relatively low, even among this sample which is likely to be more sex-positive.

This study demonstrates the importance of sexual communication self-efficacy, and presents evidence supporting validity and internal reliability of a new scale. Future research 
should examine the factors which foster sexual communication self-efficacy. Research continues to demonstrate the importance of sexual socialization, particularly during adolescence. For instance, Bleakley, Hennessy, Fishbein and Jorgan (2009) found that adolescents learn about sex from their friends, mothers, media, and doctors. Interestingly, they found that relying on media for sexual information was related to beliefs that engaging in sex would have positive outcomes and, meanwhile, relying on mothers as a source of sexual information was associated with beliefs that engaging in sex would result in negative physical outcomes. Additionally, future research should incorporate the Sexual Communication Self-Efficacy Scale into predictive models with the intention of measuring actual condom use.

In sum, the goal of this study was to evaluate the factor structure, reliability, and validity of the newly developed measure of Sexual Communication Self-Efficacy. Five distinct factors emerged using maximum likelihood factor analysis: Contraceptive Communication, Negative Sexual Messages, Positive Sexual Messages, Sexual Health, and Condom Negotiation. Internal reliability of all these factors were excellent. This study also provides support for the construct validity of this scale with strong correlations in the expected directions.

\section{Implications for Practice}

This research may serve as a starting point for new interventions that aim to increase sexual health, reduce sexual risk taking, and enhance sexual relationships among young people. In particular, this study suggests that young people in the UK show deficits in sexual communication self-efficacy, particularly related to disinterest in particular sexual activities, and about condom negotiation with their partners. Practitioners seeking to increase sexual communication self-efficacy should be cognizant of these particular vulnerabilities, and aim to increase self-efficacy in these areas. These results also suggest that interventions should 
combine both the risk reduction and sexual pleasure foci. The SCSES may have utility in assessing the effectiveness of these types of interventions. 


\section{References}

Bandura, A. (1994). Self-efficacy. John Wiley \& Sons, Inc.

Bleakley, A., Hennessy, M., Fishbein, M., \& Jordan, A. (2009). How sources of sexual information relate to adolescents' beliefs about sex. American Journal of Health Behavior, 33(1), 37-48.

Byers, E. S. (2005). Relationship satisfaction and sexual satisfaction: A longitudinal study of individuals in long-term relationships. Journal of Sex Research, 42(2), 113-118.

Catania, J., Coates, T., \& Kegeles, S. (1989). Predictors of condom use and multiple partnered sex among sexually active adolescent women: Implications for AIDS-related health interventions. The Journal of Sex Research, 26, 514-524.

Davies, S. L., DiClemente, R. J., Wingood, G. M., Person, S. D., Dix, E. S., Harrington, K., ... \& Oh, K. (2006). Predictors of inconsistent contraceptive use among adolescent girls: findings from a prospective study. Journal of Adolescent Health, 39(1), 43-49.

Eaton, D. K., Kann, L., Kinchen, S., Shanklin, S., Flint, K. H., Hawkins, J., ... \& Wechsler, H. (2012). Youth risk behavior surveillance-United States, 2011. Morbidity and Mortality Weekly Report. Surveillance Summaries (Washington, DC: 2002), 61(4), 1-162.

Gore, F. M., Bloem, P. J. N., Patton, G. C., Ferguson, J., Joseph, V., Coffey, C., \& ... Mathers, C.D. Global burden of disease in young people aged 10-24 years: A systematic analysis. The Lancet 377(9783), 2093-2102.

Grossman, C., Hadley, W., Brown, L. K., Houck, C. D., Peters, A., \& Tolou-Shams, M. (2008). Adolescent sexual risk: Factors predicting condom use across the stages of change. AIDS and Behavior, 12(6), 913-922. 
Guzman, B. L., Schlehofer-Sutton, M. M., Villanueva, C. M., Stritto, M. E., Casad, B. J., \& Feria, A. (2003). Let's talk about sex: How comfortable discussions about sex impact teen sexual behavior. Journal of Health Communication, 8(6), 583-598.

Halpern-Felsher, B. L., Kropp, R. Y., Boyer, C. B., Tschann, J. M., \& Ellen, J. M. (2004). Adolescents' self-efficacy to communicate about sex: Its role in condom attitudes, commitment and use. Adolescence, 39(155), 443-456.

Health Protection Agency. (2008) Sexually transmitted infections and young people in the United Kingdom. Retrieved from http://webarchive.nationalarchives.gov.uk/20140714084352/http://www.hpa.org.uk/webc /HPAwebFile/HPAweb_C/1216022461534

Ivankovich, M., Leichliter, J., \& Douglas, J. (2013). Measurement of sexual health in the US: An inventory of nationally representative surveys and surevillance systems. Public Health Reports, 128, 62-72.

Pearson, J. (2006). Personal control, self-efficacy in sexual negotiation, and contraceptive risk among adolescents: The role of gender. Sex Roles, 54(9-10), 615-625.

Philpott, A., Knerr, W., \& Maher, D. (2006). Promoting protection and pleasure: Amplifying the effectiveness of barriers against sexually transmitted infections and pregnancy. The Lancet, 368, 2028-31

Rostosky, S. S., Dekhtyar, O., Cupp, P. K., \& Anderman, E. M. (2008). Sexual self-concept and sexual self-efficacy in adolescents: a possible clue to promoting sexual health? Journal of Sex Research, 45(3), 277-286. 
Sales, J. M., Milhausen, R. R., Wingood, G. M., DiClemente, R. J., Salazar, L. F., \& Crosby, R. A. (2008). Validation of a parent-adolescent communication scale for use in STD/HIV prevention interventions. Health Education \& Behavior, 35(3), 332-345.

Smiler, A. P., Ward, L. M., Caruthers, A., \& Merriwether, A. (2005). Pleasure, empowerment, and love: Factors associated with a positive first coitus. Sexuality Research and Social Policy, 2(3), 41-55

Sohal, H., Eldridge, S., \& Feder, G. (2007). The sensitivity and specificity of four questions (HARK) to identify intimate partner violence: a diagnostic accuracy study in general practice. BMC Family Practice, 8(1), 49.

Sonnenberg, P., Clifton, S., Beddows, S., Field, N., Soldan, K., Tanton, C., ... \& Johnson, A. M. (2013). Prevalence, risk factors, and uptake of interventions for sexually transmitted infections in Britain: findings from the National Surveys of Sexual Attitudes and Lifestyles (Natsal). The Lancet, 382(9907), 1795-1806.

Tabachnick, B. G., \& Fidell, L. S. (2012). Using multivariate statistics. Boston: Pearson.

Tolman, D. L., Striepe, M. I., \& Harmon, T. (2003). Gender matters: Constructing a model of adolescent sexual health. Journal of Sex Research, 40(1), 4-12.

Troth, A., \& Peterson, C. (2000). Factors predicting safe-sex talk and condom use in early sexual relationships. Health Communication, 12(2), 195-218.

Mercer, C. H., Tanton, C., Prah, P., Erens, B., Sonnenberg, P., Clifton, S., ... \& Johnson, A. M. (2013). Changes in sexual attitudes and lifestyles in Britain through the life course and over time: findings from the National Surveys of Sexual Attitudes and Lifestyles (Natsal). The Lancet, 382(9907), 1781-1794. 
Miller, K. S., Kotchick, B. A., Dorsey, S., Forehand, R., \& Ham, A. Y. (1998). Family communication about sex: What are parents saying and are their adolescents listening?. Family Planning Perspectives, 218-235.

Wellings, K., Jones, K. G., Mercer, C. H., Tanton, C., Clifton, S., Datta, J., ... \& Johnson, A. M. (2013). The prevalence of unplanned pregnancy and associated factors in Britain: findings from the third National Survey of Sexual Attitudes and Lifestyles (Natsal3). The Lancet, 382(9907), 1807-1816.

Wellings, K., Nanchahal, K., Macdowall, W., McManus, S., Erens, B., Mercer, C. H., ... \& Field, J. (2001). Sexual behaviour in Britain: early heterosexual experience. The Lancet, 358(9296), 1843-1850.

Widman, L., Welsh, D. P., Mcnulty, J. K., \& Little, K. C. (2006). Sexual communication and contraceptive use in adolescent dating couples. Journal of Adolescent Health, 39(6), 893-899. 